\title{
F-I 8 fluorodeoxyglucose (FDG) positron emission tomography (PET)-positive parotid incidentaloma: Prevalence and clinical significance
}

\author{
Urszula Kiendys' \\ Hamphrey Ham' \\ Wouter Bauters ${ }^{2}$ \\ Caroline Van den Broecke ${ }^{3}$ \\ Philippe Deron ${ }^{4}$ \\ Ingeborg Goethals' \\ 'Department of Nuclear Medicine; \\ ${ }^{2}$ Department of Radiology; \\ ${ }^{3}$ Department of Pathology; \\ ${ }^{4}$ Department of Head and Neck \\ Surgery, Ghent University Hospital, \\ Ghent, Belgium
}

\begin{abstract}
Aim: To evaluate the prevalence of parotid incidentaloma identified by F-18 fluorodeoxyglucose PET(-CT) scan and the clinical significance associated with this finding.

Methods: Records from all patients from January 1, 2005 to August 31, 2007 who had focal intense abnormal F-18 FDG uptake in the parotid glands without a previous history of squamous cell carcinoma in the head and neck, lymph node metastasis from an unknown primary in the head and neck region or lymphoma, were reviewed.

Results: Of the 5476 PET-CT scans performed in as many patients, focal high-grade parotid F-18 FDG uptake was observed in 18 patients (prevalence of $0.3 \%$ ). Eight patients (44\%) underwent surgery. Four of 8 patients (50\%) had benign tumors, 2 (25\%) had infectious disease and 1 patient each had metastatic disease from a melanoma and a non-Hodgkin's lymphoma.

Conclusion: F-18 FDG PET-positive parotid incidentaloma is rare. The prevalence of a PETpositive parotid incidentaloma in our study was $0.3 \%$. Clear-cut focal high-grade abnormal F-18 FDG uptake was seen in benign tumors, infectious and metastatic disease.
\end{abstract}

Keywords: F-18 FDG PET-CT scanning, incidentaloma, parotid gland, prevalence

\section{Introduction}

The normal F-18 fluorodeoxyglucose (FDG) positron emission tomography (PET) anatomy of the head and neck as well as F-18 FDG uptake in normal anatomic structures of the naso-, oro- and hypopharynx is well known. ${ }^{1,2}$ Also, PET with F-18 FDG has demonstrated utility in the detection of primary head and neck tumors, including tumors of the salivary glands, ${ }^{3,4}$ and metastases to lymph nodes in the head and neck region because uptake of the tracer is substantially higher in the tumor than in the normal tissues of the head and neck which also accumulate F-18 FDG.

Focal high-grade abnormal F-18 FDG uptake in the parotid glands unrelated to the primary disease (so-called parotid gland incidentaloma) has been observed with this imaging modality. However, the prevalence and the clinical significance of this finding is not well known. Recently, Basu and colleagues ${ }^{5}$ reported on the varying F-18 FDG uptake patterns in the parotid glands and the pathology associated with these findings. In this study, F-18 FDG uptake in the parotid glands was diffuse and (a)symmetrical in the majority of cases. Moreover, in these cases, no clear-cut pathology in the parotid glands could be demonstrated by correlative investigations. In the present study, diffuse and (a)symmetrical F-18 FDG uptake in the parotid glands was considered a physiological variant. Our aim was to evaluate the prevalence and the clinical significance of clear-cut focal high-grade abnormal F-18 FDG uptake in the parotid glands in another study population. 


\section{Methods}

\section{Subjects}

The cases for this study were retrospectively selected from a population of patients with cancer who underwent whole body F-18 FDG PET-computed tomography (CT) scanning at our institution from January 1, 2005 to August 31, 2007. F-18 FDG PET-CT examinations were performed as part of the initial staging protocol or for follow-up of oncologic disease. Patients with a history of squamous cell carcinoma in the head and neck, lymph node metastases from an unknown primary in the head and neck region or lymphoma were excluded. The clinical PET-CT reports that had a special reference to focal intense F-18 FDG accumulation in one of the parotid glands, irrespective of the CT findings, were the basis for this study. Final diagnosis was given by pathology or, in patients who did not undergo a surgical procedure, by clinical follow-up that was performed until August 31, 2008. The local ethics committee approved the retrospective analysis of patient files and images.

\section{F-I 8 FDG PET-CT scanning}

All examinations were performed according to the standard protocol at the Ghent University Hospital. The patients fasted at least four hours prior to intravenous injection of F-18 FDG (3.7 MBq/kg body weight). Blood glucose levels were checked prior to the injection of F-18 FDG and did not exceed $140 \mathrm{mg} / \mathrm{dL}$. If not contraindicated, iodinated intravenous contrast (iohexol, $350 \mathrm{mg}$ iodine/mL, GE Healthcare, Diegem, Belgium or iomeprol, $400 \mathrm{mg}$ iodine $/ \mathrm{mL}$, Bracco Imaging Europe, Waver, Belgium) was administered immediately before CT scanning. Patients were imaged with the Gemini PET-CT imaging system (Philips Co., Cleveland, OH, USA), which consists of a gadolineum oxyorthosilicate (GSO) full-ring PET scanner with $5.0 \mathrm{~mm}$ spatial resolution and a 16-slice helical CT scanner. After a 60-min uptake period, during which patients were instructed to rest silently, images were acquired. First, a CT surview $(30 \mathrm{~mA}, 120 \mathrm{kV}$, FOV $500 \mathrm{~mm}$, collimation $0.75 \mathrm{~mm}$ ) was performed from the base of the skull through the mid thigh. This was followed by a low-dose CT $(30 \mathrm{~mA}, 120 \mathrm{kV}$, FOV $600 \mathrm{~mm}, 0.5 \mathrm{~s}$ rotation time, pitch 0.9 , collimation $16 \times 1.5 \mathrm{~mm}$ ) and a high-quality CT $(150 \mathrm{~mA}, 120 \mathrm{kV}$, FOV $500 \mathrm{~mm}, 0.5 \mathrm{~s}$ rotation time, pitch of 0.9 , collimation $16 \times 1.5 \mathrm{~mm}$, slice thickness and increment $5 \mathrm{~mm}$ ) without specific breath-holding instructions and arms at the side of the torso. PET scanning was performed immediately after acquisition of the CT images, without changing the patient position. Between five and eight bed positions were used, with an acquisition time of $3 \mathrm{~min}$ per bed position. PET images were reconstructed by using an iterative 3D-RAMLA (Row Action Maximum Likelihood Algorithm) algorithm provided by the vendor. Low-dose CT data were used for attenuation correction.

\section{Image analysis}

Both uncorrected and attenuation-corrected F-18 FDG PET images were visually interpreted by an experienced nuclear medicine physician at a workstation equipped with fusion software (Syntegra, version 2.1E, Philips Co.) that enables the display of CT, PET and PET-CT images. PET findings were interpreted as demonstrating focal intense uptake of F-18 FDG (so-called parotid gland incidentaloma) in one of the parotid glands based on visual assessment. For correlation with CT, combined PET-CT images were interpreted in a consensus reading by the nuclear medicine physician and a radiologist with experience in the interpretation of $\mathrm{CT}$ images in head and neck region. Both were blind to the clinical findings.

In addition, we assessed the maximum standard F-18 FDG uptake in the lesions on the co-registered attenuation corrected PET images by drawing a manual region of interest over the sites of focally increased F-18 FDG activity. The standard uptake value (SUV) is a semi-quantitative measure used to express the relative F-18 FDG uptake. It is a measure of the local concentration of F-18 FDG determined from the PET images by selection of a volume of interest encompassing the lesion divided by the injected dose, normalized by body weight.

\section{Results}

During the study period 5476 patients underwent a whole body PET-CT scan. In 18 patients clear-cut focal high-grade abnormal F-18 FDG uptake in the parotid glands was found. Hence, the prevalence of a F-18 FDG PET-positive parotid incidentaloma was $0.3 \%$.

Of the patients with a F-18 FDG PET-positive incidentaloma, eight (44\%) were women and 10 (56\%) were men. The age range of patients with this finding was 14 to 81 years old with an average age of 59 years. Indications for PET-CT scanning were staging of (suspected) lung cancer based on abnormal findings on CT $(n=4)$, restaging of lung cancer $(\mathrm{n}=1)$, staging of mesothelioma $(\mathrm{n}=1)$, (re)staging of esophageal cancer $(n=3)$, follow-up of prostate cancer and hypernephroma $(n=1)$, follow-up of melanoma (outside the head and neck region) $(n=1)$, follow-up of abdominal infection $(n=2)$, prolonged fever and fatigue $(n=4)$, and exclusion of brain metastasis $(n=1)$ (for details see also Table 1 ). In 12 patients $(67 \%)$ the right parotid and in six $(33 \%)$ the left parotid was 


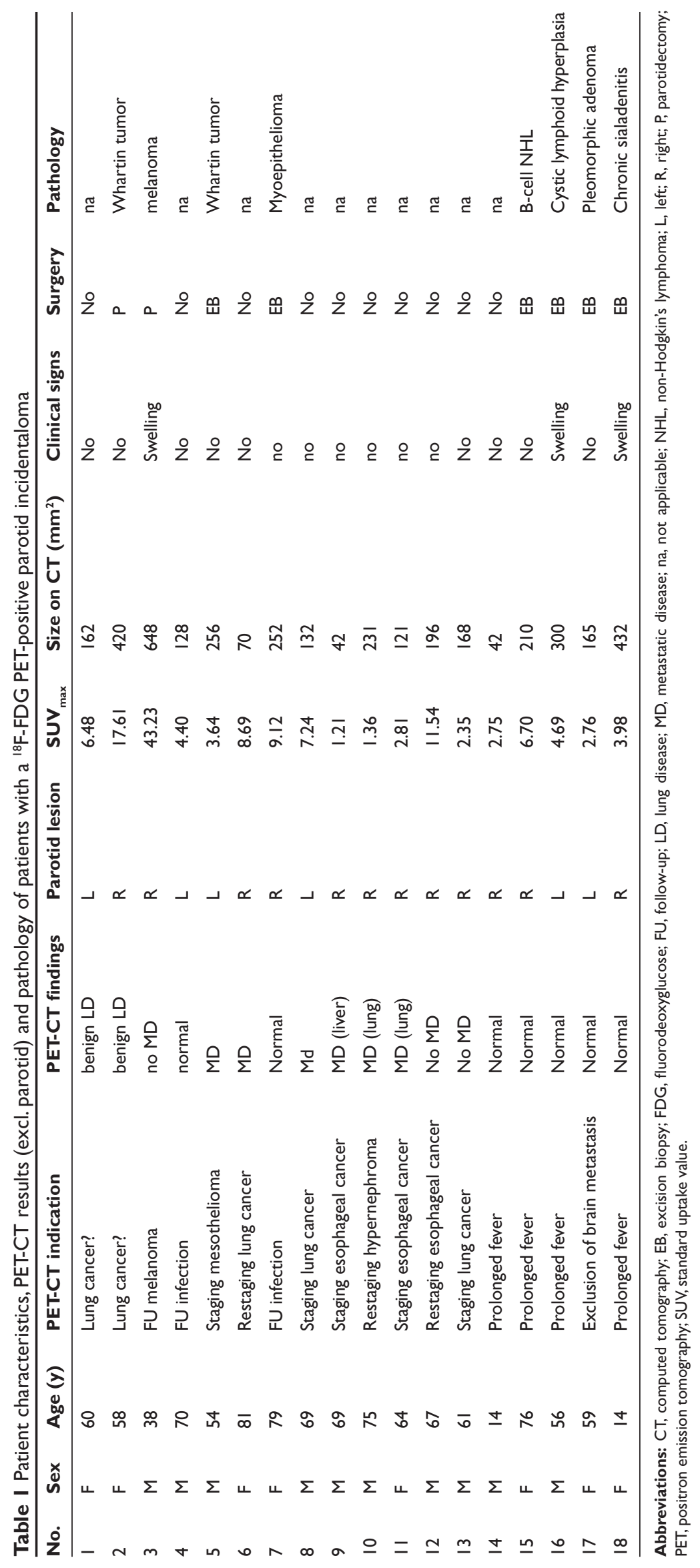


involved. Fifteen patients (83\%) were asymptomatic and three patients (17\%) had mild and painless unilateral swelling in the parotid region. In 10 patients (56\%) further diagnostic workup was not performed because of other prevailing medical conditions or because the probability of parotid malignancy was estimated low by the referring physician.

Focal high-grade F-18 FDG uptake on PET corresponded with (contrast-enhancing) nodules on the CT scan (for examples see Figures 1 and 2). The maximum two-dimensional size of the nodules varied from $42 \mathrm{~mm}^{2}$ to $648 \mathrm{~mm}^{2}$ (mean size of $221 \mathrm{~mm}^{2}$ ). On the PET scan, the maximum standard F-18 FDG uptake in the lesions ranged from 1.21 to 43.23 (median SUV of 4.55).

Eight patients (44\%) eventually underwent surgery, of whom six had an excision biopsy and two underwent superficial parotidectomy. In the patients who underwent surgical evaluation, four had benign tumors and two had infectious disease. In one patient each, metastatic disease from a melanoma and a non-Hodgkin's lymphoma was diagnosed. In the melanoma case, it is noteworthy that the primary site was outside the head and neck region, namely the right forearm, and that diagnosis was made 10 years earlier. Moreover, at the time, the sentinel node in the right axilla was free from tumor cells. In the lymphoma case, there was no previous history of lymphoma and at the time of the PET-CT scanning the parotid gland was the sole site involved. Review of the clinical charts in the patients who did not undergo a surgical procedure revealed that during the follow-up period none of them developed symptoms related to the parotid. Repeat PET-CT scan at six to nine months of follow-up was available for two patients. In one patient, focal parotid uptake had not changed both visually and semiquantitatively. In the other patient, resolution of F-18 FDG hypermetabolism was shown. Follow-up PET-CT data were not available for the remaining eight patients.
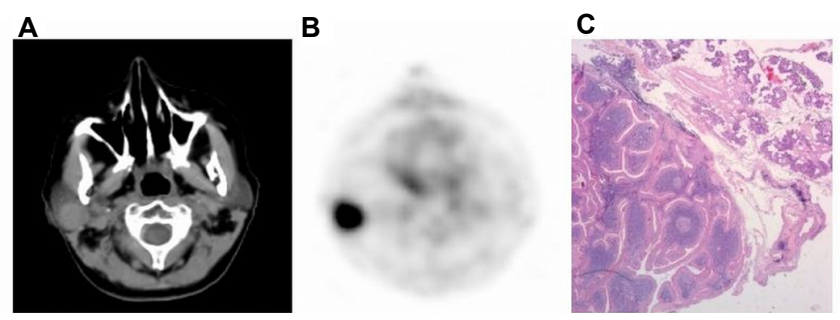

Figure I Transverse non-contrast enhanced CT (A) and F- I8 FDG PET (B) images of a right parotid mass with intense F-18 FDG uptake. On histopathology (C) a papillarycystic tumor associated with a dense lymphoid stroma was shown (Whartin tumor on the left side and normal parotid gland tissue on the right side of the image).

Abbreviations: CT, computed tomography; FDG, fluorodeoxyglucose; PET, positron emission tomography.
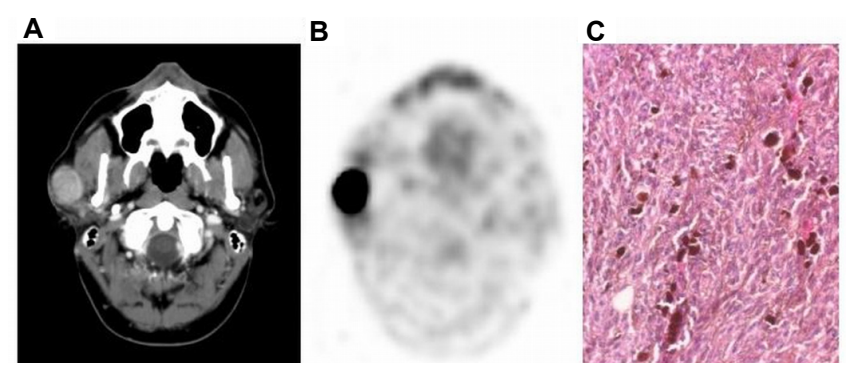

Figure 2 Transverse contrast-enhanced CT (A) and F-18 FDG PET (B) images of a right parotid mass with intense F-18 FDG uptake. On histopathology a tumor composed of spindle cells with pigmentation (melanin pigment) was shown.

Abbreviations: CT, computed tomography; FDG, fluorodeoxyglucose; PET, positron emission tomography.

F-18 FDG uptake in the lesions was not significantly different in the patients who underwent surgery and those who did not (two samples T-test, $p=0.223$ ). However, tumors that were resected were larger than those that were not (two samples T-test, $\mathrm{p}=0.007$ ).

\section{Discussion}

Over approximately a $2 \frac{1}{2}$ year-period, 5476 whole body PET-CT scans were performed in as many patients as part of the initial staging protocol or for surveillance in patients with known malignancies. In 18 patients we observed focal high-grade parotid F-18 FDG uptake that was either unexpected or unlikely related to the primary disease process. F-18 FDG PET-positive parotid incidentaloma occurred with a low incidence rate of $0.3 \%$ at our institution. This is in line with the findings of a recent study in which asymmetric focal F-18 FDG uptake in one of the parotid glands was noted in only seven patients out of 2000 PET studies reviewed. ${ }^{5}$

Eight out of 18 patients (44\%) underwent surgical evaluation. In those eight patients, we found benign tumors in four and infectious disease in two patients. Metastatic disease and a non-Hodgkin's lymphoma were found in one patient each. Our results should be viewed with caution because only $44 \%(8 / 18)$ of patients with positive parotid F-18 FDG PET findings had a biopsy or underwent resection. Because not all patients consented to surgical evaluation, the incidence of benign and malignant parotid disease in our study may not reflect the actual rate. Our results may have been skewed because of inherent selection bias in the patients who were ultimately biopsied or underwent a parotidectomy. These may have been patients whose tumors were larger and palpable or for whom the detection rate of malignant disease may be higher. Despite a probable selection bias, it is noteworthy that for those individuals who underwent surgical evaluation, no primary malignancy of the parotid 
gland was found. This finding is consistent with the fact that the majority of parotid tumors are benign. ${ }^{6}$ The most frequent histotypes, according to the new World Health Organization pathological classification, ${ }^{7}$ are pleomorphic adenoma and adenolymphoma (Whartin tumor). ${ }^{8}$ Benign tumors in our series were adenolymphoma, pleomorphic adenoma, and myoepithelioma (see also Table 1). Benign nonepithelial tumors such as angioma, lipoma, and neurogenic tumors relating to the facial nerve may also occur within the parotid gland. Metastatic disease to the parotid gland is worth considering. Metastatic involvement most commonly arises via lymphatic spread with scalp squamous cell carcinoma and melanoma the most common primaries. ${ }^{9}$ Interestingly, metastatic involvement of the parotid gland in a patient in our series was due to a 10 -years earlier diagnosed malignant melanoma outside the head and neck region. The follicular non-Hodgkin B-cell lymphoma was found in a patient who underwent whole body PET-CT scanning for prolonged fever and fatigue. At the time of the PET-CT scanning, the parotid gland was the sole site involved. Yet, on follow-up PET scanning, active disease was shown in several nodal locations. Another WHO class of salivary gland lesions are the so-called tumor-like lesions often due to both infectious and non-infectious granulomatous pathologies. Infectious granulomatous parotitis usually manifests as asymptomatic gradual painless enlargement of a nodule or diffuse enlargement of the whole parotid gland. ${ }^{10}$ In our series, chronic nonmycobacterial granulomatous sialadenitis was seen in a 14-year-old girl suffering from an autoimmune lymphoproliferative syndrome. Cystic lymphoid hyperplasia was diagnosed in a HIV-infected patient. Finally, we included in our study a patient with a parotid nodule on MRI. In this case, MRI of the head (and neck) was performed because the patient complained of atypical headaches. On MRI an intracranial mass (and a parotid nodule) was found. Subsequently, the patient underwent a whole body PET-CT scan because brain metastasis had to be excluded. PET-CT scanning was normal with the exception of focal high-grade F-18 FDG uptake in the parotid nodule. However, with abnormal F-18 FDG uptake on PET only in the parotid region, it seemed highly unlikely that the intracranial mass was a brain metastasis from a primary parotid malignancy. To obtain a definite diagnosis, the patient underwent surgery and both lesions were removed. The pathological diagnosis of the intracranial mass was a meningeoma, while the parotid nodule was a pleomorphic adenoma.

On PET images, it has become common practice to quantify tumor uptake of F-18 FDG by means of SUV.
By correcting for variations in injected dose from study to study and for patient weight, this value is expected to give a more consistent measure of tumor uptake across patient populations. Some investigators have used SUV to classify salivary gland tumors, ${ }^{3}$ and parotid masses in particular. ${ }^{4,11}$ In the report by Keyes and colleagues, ${ }^{3}$ the authors concluded that maximum and average SUV did not adequately distinguish benign lesions from malignant tumors. The high frequency of false positive results for malignancy was a major problem and was accounted for by the high uptake of F-18 FDG by Warthin tumors. Similarly, Rubello and colleagues ${ }^{11}$ concluded that ratios of left/right maximum SUV did not play a significant role in the differential diagnosis of parotid lesions. Okamura and colleagues ${ }^{4}$ demonstrated low F-18 FDG uptake and mean SUV in inflammatory lesions and high F-18 FDG accumulation in parotid carcinomas, Whartin tumors and pleomorphic adenomas. Yet, SUV was not able to differentiate malignant from benign lesions. Although the small number of patients with malignant $(\mathrm{n}=2)$ and benign $(n=6)$ lesions in our series, our findings corroborated those in the literature. SUV of malignant and benign lesions overlapped and hence had little added value for diagnosis.

\section{Conclusion}

Parotid incidentaloma detected by F-18 FDG PET(-CT) occurred with a frequency of $0.3 \%$ at our institution. Although histology was available for only eight out of 18 patients with a F-18 FDG PET-positive parotid incidentaloma, the majority of the lesions were benign. In agreement with other reports in the literature, we found that semi-quantitative values of F-18 FDG uptake could not reliably discriminate between benign and malignant parotid lesions.

\section{Disclosure}

The authors report no conflicts of interest.

\section{References}

1. Jabour BA, Choi Y, Hoh CK, et al. Extracranial head and neck: PET imaging with 2-[F-18]fluoro-2-deoxy-D-glucose and MR imaging correlation. Radiology. 1993;186:27-35.

2. Nakamoto Y, Tatsumi M, Hammoud D, Cohade C, Osman MM, Wahl RL. Normal FDG distribution patterns in the head and neck: PET/CT evaluation. Radiology. 2005;234:879-885.

3. Keyes JW Jr, Harkness BA, Greven KM, Williams DW III, Watson NE Jr, McGuirt WF. Salivary gland tumors: pretherapy evaluation with PET. Radiology. 1994;192:99-102.

4. Okamura T, Kawabe J, Koyama K, et al. Fluorine-18 fluorodeoxyglucose positron emission tomography imaging of parotid mass lesions. Acta Otolaryngol Suppl. 1998;538:209-213.

5. Basu S, Houseni M, Alavi A. Significance of incidental fluorodeoxyglucose uptake in the parotid glands and its impact on patient management. $\mathrm{Nucl}$ Med Commun. 2008;29:367-373. 
6. Licitra L, Grandi C, Prott FJ, Schornagel JH, Bruzzi P, Molinari R. Major and minor salivary glands tumors. Crit Rev Oncol Hematol. 2003; $45: 215-225$.

7. Seifert G, Sobin LH. The World Health Organization's Histological Classification of Salivary Gland Tumors. A commentary on the second edition. Cancer. 1992;70:379-385.

8. Howlett DC, Kesse KW, Hughes DV, Sallomi DF. The role of imaging in the evaluation of parotid disease. Clin Radiol. 2002;57:692-701.
9. Nuyens M, Schupbach J, Stauffer E, Zbaren P. Metastatic disease to the parotid gland. Otolaryngol Head Neck Surg. 2006;135:844-848.

10. O'Connell JE, George MK, Speculand B, Pahor AL. Mycobacterial infection of the parotid gland: an unusual cause of parotid swelling. J Laryngol Otol. 1993;107:561-564.

11. Rubello D, Nanni C, Castellucci P, et al. Does 18F-FDG PET/CT play a role in the differential diagnosis of parotid masses. Panminerva Med. $2005 ; 47: 187-189$ 